\title{
UCRL-CONF-221180
}

LAW RENCE LIVERMORE N A T IO N A L LABORATORY

\section{Development of Time Resolved X-ray Spectroscopy in High Intensity Laser-Plasma Interactions}

M. Notley, R. Weber, B. Fell, J. Jefferies, R. Freeman, A. J. Mackinnon, R. Dickson, R. Snavely, D. Hey, F. Khattak, E. Garcia Siez, G. Gregori

May 7, 2006

High Temperature Plasma Diagnostics Williamsburg, VA, United States May 11, 2006 through May 11, 2006 
This document was prepared as an account of work sponsored by an agency of the United States Government. Neither the United States Government nor the University of California nor any of their employees, makes any warranty, express or implied, or assumes any legal liability or responsibility for the accuracy, completeness, or usefulness of any information, apparatus, product, or process disclosed, or represents that its use would not infringe privately owned rights. Reference herein to any specific commercial product, process, or service by trade name, trademark, manufacturer, or otherwise, does not necessarily constitute or imply its endorsement, recommendation, or favoring by the United States Government or the University of California. The views and opinions of authors expressed herein do not necessarily state or reflect those of the United States Government or the University of California, and shall not be used for advertising or product endorsement purposes. 


\title{
Development of Time Resolved X-ray Spectroscopy in High Intensity Laser-Plasma Interactions
}

\author{
M. Notley ${ }^{1}$, R Weber $^{2}$, B Fell ${ }^{1}$, J Jefferies ${ }^{1}$, R Freeman $^{2}$, A J Mackinnon ${ }^{3}$, R Dickson ${ }^{3}$, R Snavely $^{3}$, D Hey $^{3,4}$, \\ F Khattak ${ }^{5}$, E Garcia Seiz ${ }^{5}$, D Riley ${ }^{5}$, G Gregori ${ }^{1,6}$ \\ 1 Central Laser Facility, CCLRC Rutherford Appleton Laboratory, Chilton, Oxfordshire OX11 0QX UK \\ 2 The Ohio State University, 191 West Woodruff Ave. Columbus, OH 43210 \\ 3 Lawrence Livermore National Laboratory, PO Box 808, Livermore, CA, 94551 \\ 4 University of California, Davis, CA 95616 \\ 5 Department of Mathematics and Physics, Queens University, Belfast B77 ???, UK \\ 6 Clarendon Laboratory, University of Oxford, Oxford OX1 3PU, UK
}

This paper discusses the design of a novel time-resolved Von-Hamos Bragg spectrometer to provide spectra in the region around the titanium He- $\alpha$ line. The instrument consists of a highly oriented pyrolitic graphite (HOPG) mosaic crystal coupled to a picosecond x-ray streak camera. Measurements of the time dependent behaviour from $\mathrm{Ti}$ foils illuminated with intense laser pulses can be used to improve the understanding of recombination dynamics, electron transport and phase transitions in strongly coupled dense plasma. This is important for the modelling of the compression phase in Inertial Confinement Fusion research and the study of astrophysical environments.

\section{Introduction}

X-ray spectroscopy is a technique that has been often applied to investigate radiation transport in warm dense matter (WDM) resulting from high intensity laser matter interaction experiments [1-4]. Such dense matter states are also found to be strongly coupled (i.e., with the Coulomb interaction among charged particles being dominant over their kinetic energy) and in highly transient regimes. This poses severe limits in the microscopic modelling of such systems as the usual plasma expansion methods are not applicable [5]. On the other hand, the understanding of recombination dynamics, electron transport and phase transitions in WDM regimes is important for both the modelling of extreme astrophysical environments (such as the interior of white dwarfs and neutron star atmospheres) and for the success of inertial confinement fusion (ICF) [6,7]. In this respect, the possibility of obtaining time resolved measurements is critical in such transient states. In the past, time resolved measurements at laser illuminations in the $100 \mathrm{TW}$ to $1 \mathrm{PW}$ regimes have been often limited by the large background (i.e., bremsstrahulung) noise generated during the interaction. In this paper, we present a novel instrument design which consists of a highly oriented pyrolitic graphite (HOPG) mosaic crystal coupled to a picosecond $\mathrm{x}$-ray streak camera. We will show that by combining a highly reflective HOPG crystal in a Von-Hamos configuration [8], good signal-to-noise ratio can be achieved in time-resolved $\mathrm{x}$-ray measurements resulting from high intensity laser-plasma experiments.

\section{Experimental Setup}

The experiments were carried out at the $100 \mathrm{TW}$ Vulcan laser facility at the Rutherford Appleton Laboratory (UK) using either laser pulses at 1053 $\mathrm{nm} 1.5 \mathrm{ps}$ duration, or at $527 \mathrm{~nm}$ with $1 \mathrm{~ns}$ pulse duration. These pulses were focused to $10 \mu \mathrm{m}$ and $100 \mu \mathrm{m}$ respectively to irradiate $1 \mathrm{~mm} \times 1 \mathrm{~mm}$ bare $\mathrm{Ti}$ or sandwiched $\mathrm{Al} / \mathrm{Ti} / \mathrm{Al}(2 \mu \mathrm{m}$ thick $\mathrm{Al}$ and $5 \mu \mathrm{m}$ thick Ti) foil targets. The layout of the experiment is shown in Fig 1.

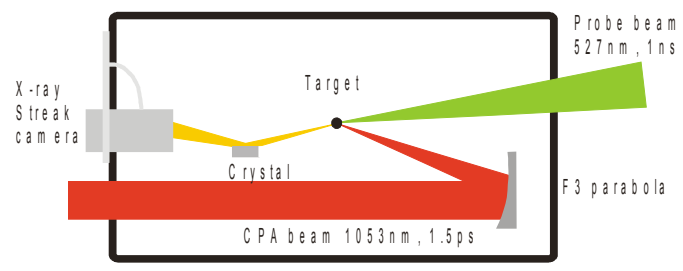

Fig 1 Experiment layout. Long pulse beam at $527 \mathrm{~nm}$ focused to $100 \mu \mathrm{m}$ using a $\mathrm{F} / 10$ lens with a random phase plate, and short pulse beam at $1053 \mathrm{~nm}$ beam focused to $10 \mu \mathrm{m}$ using a F/3 parabola.

The dispersive element of the spectrometer is ZYA grade HOPG crystal (50 mm x $25 \mathrm{~mm})$ used in mosaic focusing mode. It was configured in a Von Hamos geometry with $115 \mathrm{~mm}$ radius of curvature along the sagittal axis (i.e., the minor axis). This was coupled with a Kentech x-ray streak camera using a CsI fluffy photocathode on a $1 \mu \mathrm{m} \mathrm{CH}$ substrate flash coated with Al. Fig 2 shows the set-up of the elements of the instrument. Adjustable lateral and transverse positions of the crystal relative to the target are available and determined by radius of curvature and centre wavelength of interest. A separate pumping system was designed allow the streak camera to be kept at $10^{-6}$ mbar independently from the main target chamber. Beryllium, $1 \mathrm{~mm}$ 
thick, $50 \mathrm{~mm}$ dia. was used for the vacuum x-ray window. A 50:40 intensifier was coupled to the output phosphor of the streak camera and detection made via an 8 bit CCD capture system. The overall magnification of this system was 0.96 from input slit to output on the intensifier.

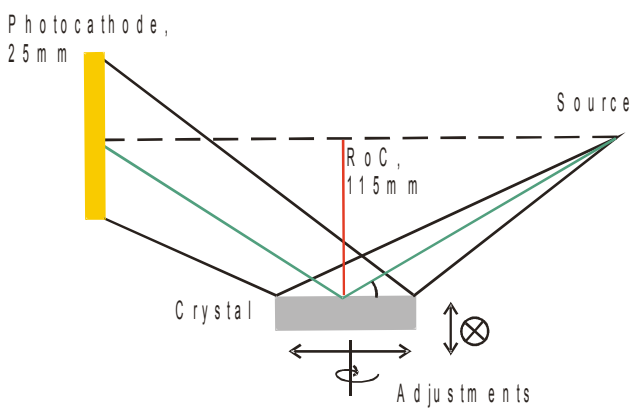

Fig 2 Spectrometer Schematic. The crystal is curved sagittally producing a line focus parallel to its surface. Detection is carried out perpendicular to this plane. The crystal can be adjusted to suit wavelength detection range. Source to cathode distance was 542 $\mathrm{mm}$

The spectrometer was designed to cover both the K$\alpha(4.5 \mathrm{keV})$ and $\mathrm{He}-\alpha$ lines $(4.75 \mathrm{keV})$ of titanium. At $\mathrm{E}_{0}=4.7 \mathrm{keV}$, the optimal source-to-crystal distance is $F=R / \sin \theta=292 \mathrm{~mm}$, where $R=115 \mathrm{~mm}$ is the radius of curvature and $\theta=23.2^{\circ}$ is the Bragg angle. The energy dispersion at the photocathode is thus [9]

$$
\frac{\Delta E}{\Delta x}=\frac{E_{0} \cos ^{2} \theta}{2 F \sin \theta}
$$

which corresponds to $\sim 17.2 \mathrm{eV} / \mathrm{mm}$ with a bandwidth of $432 \mathrm{eV}$. The spectral resolution depends both on the mosaic spread of the HOPG crystal and the geometric aberrations produced by the Von-Hamos focussing. This is estimated to be $\sim 7.1 \mathrm{eV}$ for $\mathrm{Ti}$ at $4.7 \mathrm{keV}$ [8]. Source broadening is $<2 \mathrm{eV}$ for a $100 \mu \mathrm{m}$ spot size. The time response of the fluffy CsI cathode can been estimated from Henke et al. [10]

$$
\Delta t=3.37 \frac{D^{1 / 2}}{\epsilon_{0}}\left(1+\frac{1}{4}\left(\frac{L}{S}-2\right)\left(\frac{D}{V_{0}}\right)^{1 / 2}\right),
$$

where $D$ is the energy spread (in $\mathrm{eV}$ ) of the secondary electrons emitted from the cathode, $\mathrm{V}_{0}$ is the potential between mesh and cathode $(4.1 \mathrm{kV})$ in the streak camera, $S$ the physical spacing between them $(2 \mathrm{~mm})$ and $L$ the length from mesh to the phosphors $(300 \mathrm{~mm}$ ). $\varepsilon \mathrm{o}=\mathrm{Vo} / \mathrm{S}$ (in $\mathrm{kV} / \mathrm{mm}$ ). The deposited CsI layer was 5-10 $\mu \mathrm{m}$ thick which corresponds to an energy spread of $D \sim 1.7 \mathrm{eV}$ [10]. This gives $\Delta t \sim 50-60$ ps. This number is much smaller than the time response of the HOPG crystal which is related to the time difference between rays diffracted at various depths in the crystal. This time is in the order of [11]

$$
\Delta t_{c}=\frac{2 t_{e}}{c \sin \theta},
$$

where $t_{\mathrm{e}}$ is the extinction depth $(200 \mu \mathrm{m}$ for carbon $)$ and $c$ is the speed of light. This gives $\Delta t_{c} \sim 3 \mathrm{ps}$. While a time resolution of $50 \mathrm{ps}$ is not sufficient to resolve the initial fast dynamics associated to a short-pulse laser illumination ( $\sim$ a few ps), the emission from the exploding foil and the recombining plasma develops on much longer time scales and it can still be observed. For the majority of data the streak camera was run on a time window of $1.9 \mathrm{~ns}$ (47.5 ps/mm on the phosphor) so the fastest event visible would equate to $\sim 1-1.5 \mathrm{~mm}$ on the CCD. Clearly, higher time resolutions could have been achieved by using different photocathode materials [10]. On the other hand, fluffy CsI provides the highest $\mathrm{x}$-ray sensitivity in the region of interest, and it is thus preferable for weaker signals and for studies of the recombination dynamics at late times.

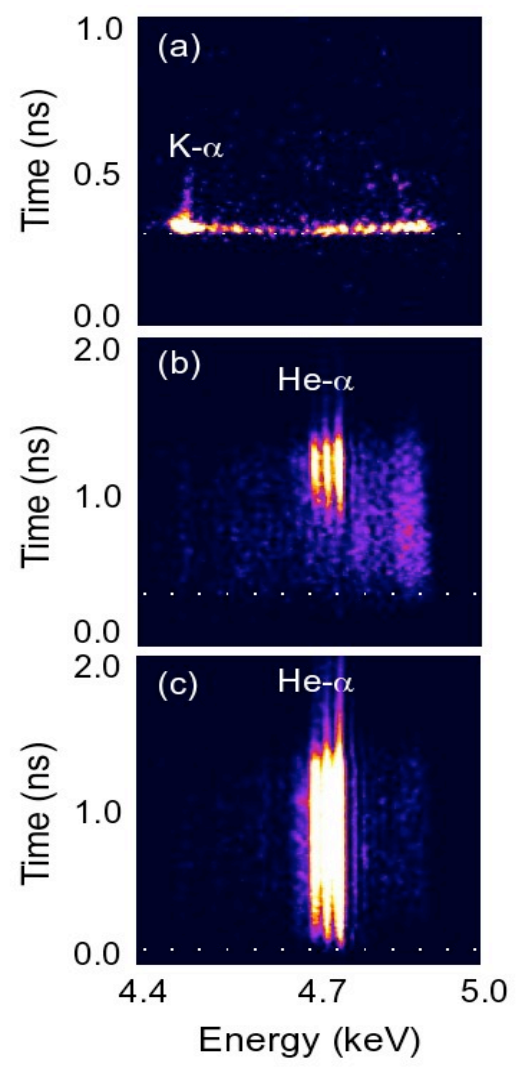

Fig 3 Experimental results. a) Streak image obtained from a $1 \times 1 \mathrm{~mm}$ layered $\mathrm{Al} / \mathrm{Ti} / \mathrm{Al}$ foil target illuminated with $125 \mathrm{~J}$ with 1.5 ps pulse at $1053 \mathrm{~nm}$; b) same target as before illuminated using $80 \mathrm{~J}$ with $1 \mathrm{~ns}$ pulse at $527 \mathrm{~nm}$; c) bare $5 \mu \mathrm{m}$ thick Ti foil illuminated at $90 \mathrm{~J}$ with a $1 \mathrm{~ns}$ pulse at $527 \mathrm{~nm}$. For all cases, the streak camera was filtered with a $25 \mu \mathrm{m} \mathrm{Al}$ foil plus a $26 \mu \mathrm{m}$ mylar foil in addition to the $1 \mathrm{~mm}$ Be window. The dotted lines indicates the start of the laser pulse. 


\section{Results}

Streaked images of the x-ray emission from both bare and sandwiched $\mathrm{Ti}$ foils, in the region between 4.4 and $5.0 \mathrm{keV}$, are shown in Fig. 3. For the case of short-pulse illumination (Fig. 3a), Ti K- $\alpha$ emission is clearly visible for about 150 ps after the start of the pulse. On the other hand, in the case of a long pulse illumination on the same target (see Fig. $3 b$ ), the only significant emission comes from the $\mathrm{He}-\alpha$ line and satellites. This starts about $\sim 0.7 \mathrm{~ns}$ after the beginning of the laser drive, indicating the time spent to burn through the Al layer [12]. Indeed, in the case of bare a Ti foil, the He- $\alpha$ emission starts immediately at the beginning of the drive pulse.

Inhomogeneities in the CsI photocathode are clearly visible at the highest streak speed (Fig. 1a). These results from a non-uniform deposition of the CsI on the $\mathrm{CH}$ substrate. Despite such effects, the K- $\alpha$ emission line remains prominent. The separation between the Ti He- $\alpha 2 p^{1} \mathrm{P}$ resonance (at $4.75 \mathrm{keV}$ ) and the $2 \mathrm{p}^{3} \mathrm{P}$ (at $4.73 \mathrm{keV}$ ) is, from Fig. 3b-c, 1.2 $\mathrm{mm}$, giving a dispersion of $19.3 \mathrm{eV} / \mathrm{mm}$ in reasonable agreement with the theoretical value. The full width at half maximum of the $2 \mathrm{p}^{1} \mathrm{P}$ line is $\sim 11 \mathrm{eV}$, slightly larger than the predicted value, indicating the possibility of source broadening.

\section{Conclusions}

We have measured temporally resolved x-ray spectra in the region from 4.4 to $5.0 \mathrm{keV}$ from $\mathrm{Ti}$ foils illuminated with intense laser pulses at the 100 TW Vulcan laser facility. These results have been obtained by coupling a high efficiency Bragg crystal to a picosecond streak camera. In order to maximize the x-ray throughput, the HOPG crystal was curved in a Von-Hamos geometry. Additional increase in sensitivity was obtained by using a CsI photocathode. We have shown that the temporal resolution of the photocathode is sufficient to observe late time $\mathrm{K}-\alpha$ emission which was observed to last for about $150 \mathrm{ps}$ after the start of the laser pulse. Our results indicates that this diagnostic can be used to study recombination dynamics, electron transport and phase transitions in WDM and for conditions relevant to ICF research.

This work was partially funded by the US DOE by the University of California Lawrence Livermore National Laboratory under contract no. W-7405ENG-48 and by the US Office of Fusion Energy Science. The work of FK, EGS and DR was funded by EPSRC grant no. xxxx.

\section{References}

[1] P. Audebert et al., Phys. Rev. Lett., 94, 025004 , (2005)

[2] K. Eidmann et al., J. Quant. Spectrosc. Radiat. Transfer, 65, 173 (2000)

[3] S.B. Hansen et al., Phys. Rev. E, submitted, (2005)

[4] G. Gregori et al., Contrib. Plasma Phys. 45, 284 (2005)

[5] S. Ichimaru, Basic Principles of Plasma Physics, Addison, Reading, MA, 1973

[6] J.D. Lindl, Inertial Confinement Fusion, Springer-Verlag, New York, 1998

[7] R.W. Lee et al., J. Opt. Soc. Am. B, 20, 770, 2003

[8] G.E. Ice and C.J. Sparks, Nucl. Instrum. Methods Phys. Res. A, 291, 110, 1990

[9] A. Pak et al., Rev. Sci. Instrum., 75, 2004

[10] B.L. Henke et al., J. Appl. Phys. 52(3), 1981

[11] Missalla et al., Rev. Sci. Instrum., 70, 1999

[12] M.H. Key et al., Phys. Fluids, 26, 2016 